\title{
Straight quantum layer with impurities inducing resonances
}

\author{
Sylwia Kondej \\ Institute of Physics, University of Zielona Góra, ul. Szafrana 4a, 65246 \\ Zielona Góra, Poland \\ e-mail: skondej@if.uz.zgora.pl,
}

\begin{abstract}
We consider a straight three dimensional quantum layer with singular potential supported on a straight wire which is localized perpendicularly to the walls and connects them. We prove that the infinite number of embedded eigenvalues appears in this system. Furthermore, we show that after introducing a small surface impurity to the layer, the embedded eigenvalues turn to the second sheet resolvent poles which state resonances. We discuss the asymptotics of the imaginary component of the resolvent pole with respect to the surface area.
\end{abstract}

Keywords: Singular perturbations, embedded eigenvalues, resonances.

\section{Introduction}

The paper belongs to the line of research often called Schrödinger operators with delta potentials 1 . The analysis of these type of potentials is motivated by mesoscopic physics systems with the semiconductor structures designed in such a way that they can be mathematically modelled by the Dirac delta supported on the sets of lower dimensions. The support of delta potential

\footnotetext{
${ }^{1}$ In the following we will equivalently use the notations delta interaction and delta potential.
} 
imitates the geometry of the semicondutor material, for example, it can take a form of one dimensional sets (wires) or surfaces with specific geometrical properties. A particle is confined in the semiconductor structure however the model admits a possibility of tunneling. Therefore these types of systems are called in literature leaky quantum graphs or wires. One of the most appealing problem in this area is the question how the geometry of a wire affects the spectrum; cf. [1] and [13, Chap. 10]. The aim of the present paper is to discuss how the surface perturbation leads to resonances.

We consider a non relativistic three dimensional model of quantum particle confined between two infinite unpenetrable parallel walls which form a straight quantum layer defined by $\Omega:=\left\{\left(\underline{x}, x_{3}\right) \in \mathbb{R}^{2} \times[0, \pi]\right\}$. In the case of absence of any additional potential the Hamiltonian of such system is given by the negative Laplacian $-\Delta: \mathrm{D}(\Delta): \rightarrow L^{2}(\Omega)$ with the domain $\mathrm{D}(\Delta)=W^{2,2}(\Omega) \cap W_{0}^{1,2}(\Omega)$, i.e. with the Dirichlet boundary conditions on $\partial \Omega$. The spectrum of $-\Delta$ is determined by $\sigma_{\text {ess }}(-\Delta)=[1, \infty)$ however it is useful to keep in mind that the energies in $x_{3}$-direction are quantized and given by $\left\{k^{2}\right\}_{k=1}^{\infty}$.

At the first stage we introduce a straight wire $I$ which connects the walls $\partial \Omega$ being at the same time perpendicular to them. We assume the presence of interaction localized on $I$ and characterized by the coupling constant $\alpha \in \mathbb{R}$. The symbolic Hamiltonian of such system can be formally written

$$
-\Delta+\delta_{\alpha, I}
$$

where $\delta_{\alpha, I}$ represents delta potential supported on $I$. Since the interaction support in this model has the co-dimension larger then one it is called strongly singular potential. The proper mathematical definition of Hamiltonian can be formulated in the terms of boundary conditions. More precisely, we define $H_{\alpha}$ as a self adjoint extension of $-\left.\Delta\right|_{C_{0}^{\infty}(\Omega \backslash I)}$ determined by means of the appropriate boundary conditions which functions from the domain $\mathrm{D}\left(H_{\alpha}\right)$ satisfy on $I$. The coupling constant $\alpha$ is involved in the mentioned boundary conditions, however, it is worth to say at this point that $\alpha$ does not contribute additively to the structure of Hamiltonian.

To describe spectral properties of $H_{\alpha}$ we can rely on radial symmetry of the system and consider two dimensional system with point interaction governed by the Hamiltonian $H_{\alpha}^{(1)}$. The spectrum of $H_{\alpha}^{(1)}$ consists of positive half line and one discrete negative eigenvalue

$$
\xi_{\alpha}=-4 \mathrm{e}^{2(-2 \pi \alpha+\psi(1))},
$$




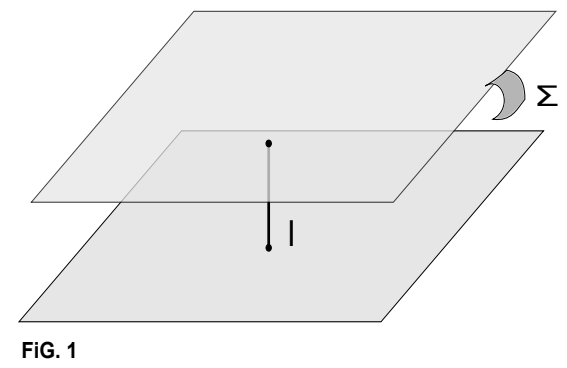

cf. [1], where $-\psi(1)=0,577 \ldots$ determines the Euler-Mascheroni constant. This reflexes the structure of spectrum of $H_{\alpha}$, namely, for each $l \in \mathbb{N}$ the number

$$
\epsilon_{l}=\xi_{\alpha}+l^{2}
$$

gives rise to an eigenvalue of $H_{\alpha}$. Note that infinite number of $\epsilon_{l}$ lives above the threshold of the essential spectrum and, consequently, the Hamiltonian $H_{\alpha}$ admits the infinite number of embedded eigenvalues.

In the second stage we introduce to the layer an attractive interaction supported on a finite $C^{2}$ surface $\Sigma \subset \Omega$ separated from a wire $I$ by some distance, cf. Fig. 1. Suppose that $\beta \neq 0$ is a real number. The Hamiltonian $H_{\alpha, \beta}$ which governs this system can be symbolically written as

$$
-\Delta+\delta_{\alpha, I}-\beta \delta_{\Sigma}
$$

where $\delta_{\Sigma}$ stands for the Dirac delta supported on $\Sigma$; this term represents weakly singular potential Again, a proper mathematical definition of $H_{\alpha, \beta}$ can be formulated as a self adjoint extension of

$$
\left.H_{\alpha}\right|_{\left\{f \in \mathrm{D}\left(H_{\alpha}\right) \cap C^{\infty}(\Omega \backslash I): f=0 \text { on } \Sigma\right\}} .
$$

This extension is defined by means of the appropriate boundary conditions on $\Sigma$ discussed in Section 3 .

The aim of this paper is to analyse how the presence of surface interaction supported on $\Sigma$ affects the embedded eigenvalues. The existence of embedded eigenvalues is a direct consequence of the symmetry. By introducing additional interaction on $\Sigma$ we break this symmetry, however, if the perturbation is small then we may expect that the system preserves a "spectral memory " on original eigenvalues. In Section 5 we show, for example, that if the area $|\Sigma| \rightarrow 0$ then the embedded eigenvalues $\epsilon_{l}$ turn to complex poles of the resolvent of $H_{\alpha, \beta}$. These poles are given by $z_{l}=\epsilon_{l}+o(|\Sigma|)$ with $\Im z_{l}<0$; 
the latter confirms that $z_{l}$ is localized on the second sheet continuation. We derive the explicit formula for the lowest order of the imaginary component of $z_{l}$ and show that it admits the following asymptotics

$$
\Im z_{l}=\mathcal{O}\left(|\Sigma|^{2}\right)
$$

The poles of resolvent state resonances in the system governed by $H_{\alpha, \beta}$ and $\Im z_{l}$ is related to the width of the resonance given by $-2 \Im z_{l}$.

Finally, let us mention that various types of resonators in waveguides and layers have been already analyzed. For example, in 22 the authors study resonances induced by the twisting of waveguide which is responsible for breaking symmetry. The planar waveguide with narrows playing the role of resonators has been studied in [4]. On the other hand, the straight Dirichlet or Neuman waveguides with windows or barriers inducing resonances have been analyzed in [6, 16, 17]. Furthermore, resonances in the curved waveguides with finite branches have been described in [10]. It is also worth to mention that quantum waveguides with electric and magnetic fields have been considered, cf. [3, 8].

On the other hand, the various types of resonators induced by delta potential in two or three dimensional systems have been analyzed. Let us mention the results of [12, 19, 20] which describe resonances in the terms of breaking symmetry parameters or by means of tunnelling effect.

In [21] the authors consider a straight two dimensional waveguide with a semitransparent perpendicular barrier modeled by delta potential. It was shown that after changing slightly the slope of barrier the embedded eigenvalues turn to resonances; the widths of these resonances can be expressed in the terms of the barrier slope. The present paper is, in a sense, an extension of [21]. However, the strongly singular character of the delta interaction supported on $I$ causes that even an infinitesimal change of the slope of $I$, can not be understood as a small perturbation. Therefore the resolvent poles are not interesting from the physical point of view since they rapidly escape far away from the real line. In the present model the role of small perturbation plays delta potential on $\Sigma$ which leads to resonances.

Finally, it is worth to mention that the spectral properties of quantum waveguides and layers with delta interaction have been studied, for example, in [14, 15]. The results of [14] concern weakly singular potentials and in [15] the authors consider strongly singular interaction. In the present paper we 
combine both types of delta interaction and analyze how they affect each other.

General notations:

- $\mathbb{C}$ stands for the complex plane and $\mathbb{C}_{ \pm}$for the upper, respectively, lower half-plane.

- $\|\cdot\|,(\cdot, \cdot)$ denote the norm and the scalar product in $L^{2}(\Omega)$ and $(\cdot, \cdot)_{\Sigma}$ defines the scalar product in $L^{2}(\Sigma)$.

- Suppose that $A$ stand for a self adjoint operator. We standardly denote by $\sigma_{\text {ess }}(A), \sigma_{\mathrm{p}}(A)$ and $\rho(A)$, respectively, the essential spectrum, the point spectrum and the resolvent set of $A$.

- The notation $C$ stands for a constant which value can vary from line to line.

\section{Parallel walls connected by wire inducing embedded eigenvalues}

\subsection{Free particle in layer.}

Let $\Omega \subset \mathbb{R}^{3}$ stand for a layer defined by $\Omega:=\left\{x=\left(\underline{x}, x_{3}\right): \underline{x} \in \mathbb{R}^{2}, x_{3} \in\right.$ $[0, \pi]\}$ and in the following we assume convention $\underline{x}=\left(x_{1}, x_{2}\right) \in \mathbb{R}^{2}$.

The "free" Hamiltonian is determined by

$$
H=-\Delta: \mathrm{D}(H)=W^{2,2}(\Omega) \cap W_{0}^{1,2}(\Omega) \rightarrow L^{2}(\Omega)
$$

and it admits the following decomposition

$$
H=-\Delta^{(2)} \otimes I+I \otimes-\Delta^{(1)} \quad \text { on } \quad L^{2}\left(\mathbb{R}^{2}\right) \otimes L^{2}(0, \pi),
$$

where $\Delta^{(2)}: \mathrm{D}\left(\Delta^{(2)}\right)=W^{2,2}\left(\mathbb{R}^{2}\right) \rightarrow L^{2}\left(\mathbb{R}^{2}\right)$ stands for the two-dimensional Laplacian and $\Delta^{(1)}: \mathrm{D}\left(\Delta^{(1)}\right)=W^{2,2}(0, \pi) \cap W_{0}^{1,2}(0, \pi) \rightarrow L^{2}(0, \pi)$ determines one-dimensional Laplacian with the Dirichlet boundary conditions.

To define the resolvent of $H$ it is useful to note that the sequence $\left\{\chi_{n}\right\}_{n=1}^{\infty}$ given by

$$
\chi_{n}\left(x_{3}\right):=\sqrt{\frac{2}{\pi}} \sin \left(n x_{3}\right), \quad n \in \mathbb{N}
$$


forms an orthonormal basis in $L^{2}(0, \pi)$. Suppose that $z \in \mathbb{C} \backslash[1, \infty)$. Then $R(z):=(-\Delta-z)^{-1}$ defines an integral operator with the kernel

$$
\mathcal{G}\left(z ; \underline{x}, \underline{x}^{\prime}, x_{3}, x_{3}^{\prime}\right):=\frac{1}{2 \pi} \sum_{n=1}^{\infty} K_{0}\left(\kappa_{n}(z)\left|\underline{x}-\underline{x}^{\prime}\right|\right) \chi_{n}\left(x_{3}\right) \chi_{n}\left(x_{3}^{\prime}\right),
$$

where $K_{0}(\cdot)$ denotes the Macdonald function, cf. [2], and

$$
\kappa_{n}(z):=-i \sqrt{z-n^{2}}, \quad \Im \sqrt{z-n^{2}}>0 .
$$

In the following we will also use the abbreviation $\mathcal{G}(z)$ for $(2.3)$. The threshold of spectrum of $H$ is determined by the lowest discrete transversal energy, i.e. 1. Moreover, it is purely absolutely continuous and consequently, it takes the form

$$
\sigma(H)=[1, \infty)
$$

\subsection{Layer with perpendicular wire: embedded eigen- values phenomena.}

We introduce a wire defined by the straight segment of width $\pi$ and perpendicular to walls. The presence of the wire will be modelled by delta interaction supported on $I \subset \Omega$, where $I:=(0,0) \times[0, \pi]$.

In view of the radial symmetry the operator with delta interaction on $I$ admits a natural decomposition on $L^{2}(\Omega)=L^{2}\left(\mathbb{R}^{2}\right) \otimes L^{2}(0, \pi)$ and acts in the subspace $L^{2}\left(\mathbb{R}^{2}\right)$ as the Schrödinger operator with one point interaction. Therefore, the delta potential can be determined by appropriate boundary conditions, cf. [1, Chap. 1.5], which can be implemented, in each sector of the transversal energy, separately. For this aim we decompose a function $\psi \in$ $L^{2}(\Omega)$ onto $\psi(x)=\sum_{n=1}^{\infty} \psi_{n}(\underline{x}) \chi_{n}\left(x_{3}\right)$, where $\psi_{n}(\underline{x}):=\int_{0}^{\pi} \psi\left(\underline{x}, x_{3}\right) \chi_{n}\left(x_{3}\right) \mathrm{d} x_{3}$.

$\left.D_{1}\right)$ We say that a function $\psi$ belongs to the set $D^{\prime} \subset W_{\text {loc }}^{2,2}(\Omega \backslash I) \cap L^{2}(\Omega)$ if $\Delta \psi \in L^{2}(\Omega),\left.\psi\right|_{\partial \Omega}=0$ and the following limits

$$
\Xi_{n}(\psi):=-\lim _{|\underline{x}| \rightarrow 0} \frac{1}{\ln |\underline{x}|} \psi_{n}(\underline{x}), \quad \Omega_{n}(\psi):=\lim _{|\underline{x}| \rightarrow 0}\left(\psi_{n}(|\underline{x}|)-\Xi_{n}(\psi) \ln |\underline{x}|\right)
$$

are finite. 
$\left.D_{2}\right)$ For $\alpha \in \mathbb{R}$, we define the set

$$
\mathrm{D}\left(H_{\alpha}\right):=\left\{\psi \in D^{\prime}: 2 \pi \alpha \Xi_{n}(\psi)=\Omega_{n}(\psi) \text { for any } n \in \mathbb{N}\right\}
$$

and the operator $H_{\alpha}: \mathrm{D}\left(H_{\alpha}\right) \rightarrow L^{2}(\Omega)$ which acts

$$
H_{\alpha} \psi(x)=-\Delta \psi(x), \quad \text { for } \quad x \in \Omega \backslash I .
$$

The resulting operator $H_{\alpha}: D\left(H_{\alpha}\right) \rightarrow L^{2}(\Omega)$ coincides

$$
-\Delta_{\alpha}^{(2)} \otimes I+I \otimes-\Delta^{(1)} \quad \text { on } \quad L^{2}\left(\mathbb{R}^{2}\right) \otimes L^{2}(0, \pi),
$$

where $\Delta_{\alpha}^{(2)}: \mathrm{D}\left(\Delta_{\alpha}^{(2)}\right) \rightarrow L^{2}\left(\mathbb{R}^{2}\right)$ stands for the two-dimensional Laplacian with point interaction, cf. [1, Chap. 1.5] with the domain $\mathrm{D}\left(\Delta_{\alpha}^{(2)}\right)$. Consequently, $H_{\alpha}$ is self adjoint and its spectral properties will be discussed in the next section.

\subsection{Resolvent of $H_{\alpha}$.}

Suppose that $z \in \mathbb{C}_{+}$. We use the standard notation $R_{\alpha}(z)$ for the resolvent operator, i.e. $R_{\alpha}(z):=\left(H_{\alpha}-z\right)^{-1}$. To figure out the explicit resolvent formula we introduce

$$
\omega_{n}(z ; x):=\frac{1}{2 \pi} K_{0}\left(\kappa_{n}(z)|\underline{x}|\right) \chi_{n}\left(x_{3}\right), \quad n \in \mathbb{N} ;
$$

in the following we will use also abbreviation $\omega_{n}(z)=\omega_{n}(z ; \cdot)$.

The following theorem states the desired result.

Theorem 2.1 The essential spectrum of $H_{\alpha}$ is given by

$$
\sigma_{\text {ess }}\left(H_{\alpha}\right)=[1, \infty) .
$$

Furthermore, let ${ }^{2}$

$$
\Gamma_{n}(z):=\frac{1}{2 \pi}\left(2 \pi \alpha+s_{n}(z)\right), \quad \text { where } \quad s_{n}(z):=-\psi(1)+\ln \frac{\sqrt{z-n^{2}}}{2 i} .
$$

\footnotetext{
${ }^{2}$ Analogously as in the previous discussion we assume $\Im \sqrt{z-n^{2}}>0$. The logarithmic function $z \mapsto \ln z$ is defined in the cut plane $-\pi<\arg z<\pi$ and admits continuation to entire logarithmic Riemann surface.
} 
Suppose that $z \in \mathbb{C} \backslash[1, \infty)$ and $\Gamma_{n}(z) \neq 0$. Then $z \in \rho\left(H_{\alpha}\right)$ and operator $R_{\alpha}(z)$ admits the Krein-like form:

$$
R_{\alpha}(z)=R(z)+\sum_{n=1}^{\infty} \Gamma_{n}(z)^{-1}\left(\omega_{n}(\bar{z}), \cdot\right) \omega_{n}(z) .
$$

Proof. Our first aim is to show that 2.9 defines the resolvent of $H_{\alpha}$. Operator $H_{\alpha}$ is defined as the self adjoint extension of $-\left.\Delta\right|_{C_{0}^{\infty}(\Omega \backslash I)}$. Suppose that $f \in C_{0}^{\infty}(\Omega \backslash I)$. Then $g:=(-\Delta-z) f \in C_{0}^{\infty}(\Omega \backslash I)$. Employing the fact that $\omega_{n}(z)=\mathcal{G}(z) *\left(\delta \chi_{n}\right)$, where $\mathcal{G}(z)$ is the kernel defined by (2.3) and $\delta=\delta(\underline{x})$, we conclude that $\left(\omega_{n}(\bar{z}), g\right)=\left\langle\delta \chi_{n}, f\right\rangle_{-1,1}=0$ where $\langle\cdot, \cdot\rangle_{-1,1}$ states the duality between $W^{-1,2}(\Omega)$ and $W^{1,2}(\Omega)$. This, consequently, implies $R_{\alpha}(z)(-\Delta-z) f=R(z)(-\Delta-z) f=f$ in view of $(2.9)$ which means that $R_{\alpha}(z)$ defines the resolvent of a self adjoint extension of $-\left.\Delta\right|_{C_{0}^{\infty}(\Omega \backslash I)}$. To complete the proof we have to show that any function $g=R_{\alpha}(z) f$ satisfies boundary conditions (2.5). In fact, $g$ admits the unique decomposition $g=g_{1}+g_{2}$, where $g_{1}:=R(z) f$ and $g_{2}=\sum_{n=1}^{\infty} \Gamma_{n}(z)^{-1}\left(\omega_{n}(\bar{z}), f\right) \omega_{n}(z)$. Therefore, a nontrivial contribution to $\Xi_{n}(g)$ comes from $g_{2}$ since $g_{1} \in W^{2,2}(\Omega)$. Employing the asymptotic behaviour of the Macdonald function, cf. [2]

$$
K_{0}(\rho)=\ln \frac{1}{\rho}+\psi(1)+\mathcal{O}(\rho)
$$

we get $\Xi_{n}(g)=\frac{1}{2 \pi} \Gamma_{n}(z)^{-1}\left(\omega_{n}(\bar{z}), f\right)$ and

$$
\Omega_{n}(g)=\left(1-\frac{1}{2 \pi} \Gamma_{n}(z)^{-1} s_{n}(z)\right)\left(\omega_{n}(\bar{z}), f\right)=\alpha \Gamma_{n}(z)^{-1}\left(\omega_{n}(\bar{z}), f\right) .
$$

Using (2.8) one obtains (2.5). This completes the proof of 2.9 . The stability of the essential spectrum can be concluded in the analogous way as in [7, Thm. 3.1]. The key step is to show that $R(z)-R_{\alpha}(z)$ is compact. The statement can be proved relying on compactness of the trace map $S: W^{2,2}(\Omega) \rightarrow L^{2}(I)$ which follows from the boundedness of the trace map, cf. [23, Chap. 1, Thm. 8.3] and the compactness theorem, cf. [23, Chap. 1, Thm. 16.1]. This implies, in view of boundedness of $R(z): L^{2}(\Omega) \rightarrow$ $W_{0}^{1,2}(\Omega) \cap W^{2,2}(\Omega)$, that $S R(z): L^{2}(\Omega) \rightarrow L^{2}(I)$ is compact. Employing the resolvent formula, cf. [24], and the fact that the remaining operators contributing to $R(z)-R_{\alpha}(z)$ are bounded we conclude that $R(z)-R_{\alpha}(z)$ is compact. 
Remark 2.2 The spectral analysis developed in this work is mainly based on the resolvent properties. In the following we will use the results of [7, 5, 24, 25] where strongly as well as weakly singular potentials were considered.

In the following theorem we state the existence of eigenvalues of $H_{\alpha}$.

Theorem 2.3 Let $\mathcal{A}_{\alpha}:=\left\{n \in \mathbb{N}: \xi_{\alpha}+n^{2}<1\right\}$. Each $\epsilon_{n}:=\xi_{\alpha}+n^{2}$, where $n \in \mathcal{A}_{\alpha}$ defines the discrete eigenvalue of $H_{\alpha}$ with the corresponding eigenfunction $\omega_{n}:=\omega_{n}\left(\epsilon_{n}\right)$. In particular, this means that for any $\alpha$ operator $H_{\alpha}$ has at least one eigenvalue $\epsilon_{1}$ below the threshold of the essential spectrum. Operator $H_{\alpha}$ has infinite number of embedded eigenvalues. More precisely, for any $n \in \mathbb{N} \backslash \mathcal{A}_{\alpha}$ the number $\epsilon_{n}:=\xi_{\alpha}+n^{2}$ determines the embedded eigenvalue. In particular, there exists $\tilde{n} \in \mathbb{N} \backslash \mathcal{A}_{\alpha}$ such that $\epsilon_{n} \in\left((n-1)^{2}, n^{2}\right)$ for any $n>\tilde{n}$.

Proof. The proof is based on the Birman-Schwinger argument which, in view of $(2.9)$, reads

$$
z \in \sigma_{\mathrm{p}}\left(H_{\alpha}\right) \quad \Leftrightarrow \quad \exists n \in \mathbb{N}: \Gamma_{n}(z)=0
$$

cf. [25, Thm. 2.2]. Note that, given $n \in \mathbb{N}$ the function $z \mapsto \Gamma_{n}(z), z \in\{\mathbb{C}$ : $\left.\Im \sqrt{z-n^{2}}>0\right\}$ has the unique zero at $z=\xi_{\alpha}+n^{2}$, i.e.

$$
\Gamma_{n}\left(\xi_{\alpha}+n^{2}\right)=0 .
$$

Finally, it follows, for example, from [25, Thm. 3.4] that the corresponding eigenfunctions takes the form $\mathcal{G}\left(\epsilon_{l}\right) * \chi_{n} \delta$. This completes the proof.

\section{$3 \quad$ Surface impurity}

We define a finite smooth parameterized surface $\Sigma \subset \Omega$ being a graph of the map $U \ni q=\left(q_{1}, q_{2}\right) \mapsto x(q) \in \Omega$. The surface element can be calculated by means of the standard formula $\mathrm{d} \Sigma=\left|\partial_{q_{1}} x(q) \times \partial_{q_{1}} x(q)\right| \mathrm{d} q$. Additionally we assume that $\Sigma \cap I=\emptyset$. Furthermore, let $n: \Sigma \rightarrow \mathbb{R}^{3}$ stand for the unit normal vector (with an arbitrary orientation) and $\partial_{n}$ denote the normal derivative defined by vector $n$. Relying on the Sobolev theorem we state that the trace map $\left.W^{1,2}(\Omega) \ni \psi \mapsto \psi\right|_{\Sigma} \in L^{2}(\Sigma)$ constitutes a bounded operator; we set the notation $(\cdot, \cdot)_{\Sigma}$ for the scalar product in $L^{2}(\Sigma)$. Given 
$\beta \in \mathbb{R} \backslash\{0\}$ we define the following boundary conditions: suppose that $\psi \in C(\Omega) \cap C^{1}(\Omega \backslash \Sigma)$ satisfies

$$
\left.\partial_{n}^{+} \psi\right|_{\Sigma}-\left.\partial_{n}^{-} \psi\right|_{\Sigma}=-\left.\beta \psi\right|_{\Sigma}
$$

where the partial derivatives contributing to the above expression are defined as the positive, resp., negative limits on $\Sigma$ and signs are understood with respect to direction of $n$.

$\left.D_{3}\right)$ We say that a function $\psi$ belongs to the set $\breve{D} \subset W_{\text {loc }}^{2,2}(\Omega \backslash(I \cup \Sigma))$ if $\Delta \psi \in L^{2}(\Omega),\left.\psi\right|_{\partial \Omega}=0$ and the limiting equations 2.5 and 3.11 are satisfied.

$D_{4}$ ) Define operator which for $f \in \breve{D}$ acts as $-\Delta f(x)$ if $x \in \Omega \backslash(I \cup \Sigma)$ and let $H_{\alpha, \beta}: \mathrm{D}\left(H_{\alpha, \beta}\right) \rightarrow L^{2}(\Omega)$ stand for its closure.

To figure out the resolvent of $H_{\alpha, \beta}$ we define the operator acting from to $L^{2}(\Omega)$ to $L^{2}(\Sigma)$ as $R_{\alpha, \Sigma}(z) f=\left.\left(R_{\alpha}(z) f\right)\right|_{L^{2}(\Sigma)}$. Furthermore, we introduce the operator from $L^{2}(\Sigma)$ to $L^{2}(\Omega)$ defined by $\mathrm{R}_{\alpha, \Sigma}(z) f=\mathcal{G}_{\alpha} * f \delta$, where $\mathcal{G}_{\alpha}$ stands for kernel of $(2.9)$. Finally, we define $\mathrm{R}_{\alpha, \Sigma \Sigma}(z): L^{2}(\Sigma) \rightarrow L^{2}(\Sigma)$ by $\mathrm{R}_{\alpha, \Sigma \Sigma}(z) f=\left.\left(\mathrm{R}_{\alpha, \Sigma}(z) f\right)\right|_{\Sigma}$. In view of $(2.9)$ the latter takes the following form

$$
\mathrm{R}_{\alpha, \Sigma \Sigma}(z)=\mathrm{R}_{\Sigma \Sigma}(z)+\sum_{n=1}^{\infty} \Gamma_{n}(z)^{-1}\left(w_{n}(\bar{z}), \cdot\right)_{\Sigma} w_{n}(z),
$$

where $w_{n}(z):=\left.\omega_{n}(z)\right|_{\Sigma}$ and $\mathrm{R}_{\Sigma \Sigma}(z): L^{2}(\Sigma) \rightarrow L^{2}(\Sigma)$ stands for the bilateral embedding of $R(z)$.

Following the strategy developed in [24] we define the set $Z \subset \rho\left(H_{\alpha}\right)$ such that $z$ belongs to $Z$ if the operators

$$
\left(I-\beta \mathrm{R}_{\alpha, \Sigma \Sigma}(z)\right)^{-1}, \quad \text { and } \quad\left(I-\beta \mathrm{R}_{\alpha, \Sigma \Sigma}(\bar{z})\right)^{-1}
$$

acting from $L^{2}(\Sigma)$ to $L^{2}(\Sigma)$ exist and are bounded. Our aim is to show that

$$
Z \neq \emptyset
$$

Therefore we auxiliary define the quadratic below bounded form

$$
\int_{\Omega}|\psi|^{2} \mathrm{~d} x-\left.\beta \int_{\Sigma}|\psi|_{\Sigma}\right|^{2} \mathrm{~d} \Sigma, \quad \psi \in W_{0}^{1,2}(\Omega) .
$$


Let $\breve{H}_{\beta}$ stand for the operator associated to the above form in the sense of the first representation theorem, cf. [18, Chap.VI]. Following the arguments from [7] we conclude that $I-\beta \mathrm{R}_{\Sigma \Sigma}(z): L^{2}(\Sigma) \rightarrow L^{2}(\Sigma)$ defines the BirmanSchwinger operator for $\breve{H}_{\beta}$. Using Thm. 2.2 of [25] one obtains

$$
z \in \rho\left(\breve{H}_{\beta}\right) \Leftrightarrow 0 \in \rho\left(I-\beta \mathrm{R}_{\Sigma \Sigma}(z)\right) .
$$

In the following we are interested in negative spectral parameter and thus we assume $z=-\lambda$ where $\lambda>0$. Since the spectrum of $\breve{H}_{\beta}$ is lower bounded we conclude

$$
0 \in \rho\left(I-\beta \mathrm{R}_{\Sigma \Sigma}(-\lambda)\right),
$$

for $\lambda$ large enough.

Next step is to find a bound for the second component contributing to (3.12). In fact, it can be majorized by

$$
\sum_{n=1}^{\infty}\left|\Gamma_{n}(-\lambda)^{-1}\right|\left\|w_{n}(-\lambda)\right\|_{\Sigma}^{2} \leq C \sum_{n=1}^{\infty}\left\|w_{n}(-\lambda)\right\|_{\Sigma}^{2},
$$

where we applied the uniform bound $\left|\Gamma_{n}(-\lambda)^{-1}\right| \leq C$, cf. (2.8). Using the large argument expansion, cf. [2],

$$
K_{0}(z) \sim \sqrt{\frac{\pi}{2 z}} \mathrm{e}^{-z}
$$

we get the estimate

$$
\left|w_{n}(-\lambda, x)\right| \leq C \frac{1}{\lambda^{1 / 4}} \mathrm{e}^{-r_{\min }\left(n^{2}+\lambda\right)^{1 / 2}} \quad \text { for } \lambda \rightarrow \infty,
$$

where $r_{\min }=\min _{x \in \Sigma}|\underline{x}|$. This implies that the norm of the second component of (3.12) behave as $o\left(\lambda^{-1}\right)$. Combining this result with (3.14) we conclude that $0 \in \rho\left(I-\beta \mathrm{R}_{\alpha, \Sigma \Sigma}(-\lambda)\right)$ for $\lambda$ sufficiently large which shows that (3.13) holds.

To realize the strategy of [24] we observe that the embedding operator $\tau^{*}: L^{2}(\Sigma) \rightarrow W^{-1,2}(\Omega)$ acting as $\tau^{*} f=f * \delta$ is bounded and, moreover,

$$
\operatorname{Ran} \tau^{*} \cap L^{2}(\Omega)=\{0\} .
$$

Suppose that $z \in Z$. Using (3.16) together with Thm. 2.1 of [24] we conclude that the expression

$$
R_{\alpha, \beta}(z)=R_{\alpha}(z)+\mathrm{R}_{\alpha, \Sigma}(z)\left(I-\beta \mathrm{R}_{\alpha, \Sigma \Sigma}(z)\right)^{-1} R_{\alpha, \Sigma}(z) .
$$

defines the resolvent of self adjoint operator. 
Theorem 3.1 We have

$$
R_{\alpha, \beta}(z)=\left(H_{\alpha, \beta}-z\right)^{-1} .
$$

Proof. To show the statement we repeat the strategy applied in the proof of Theorem 2.1. Operator $H_{\alpha, \beta}$ is defined as the self adjoint extension of $-\left.\Delta\right|_{C_{0}^{\infty}(\Omega \backslash(I \cup \Sigma))}$ determined by imposing boundary conditions $(2.5)$ and (3.11). The idea is show that any function from the domain $\mathrm{D}\left(H_{\alpha, \beta}\right)$ satisfies (2.5) and (3.11). Since the proof can be done by the mimicking the arguments from the proof of Theorem 2.1] we omit further details.

Furthermore, repeating the arguments from the proof of Theorem 2.1 we state that

$$
\sigma_{\text {ess }}\left(H_{\alpha, \beta}\right)=[1, \infty) .
$$

Notation. In the following we will be interested in the spectral asymptotic for small $|\Sigma|$. Therefore, we introduce an appropriate scaling with respect to a point $x_{0} \in \Sigma$. Namely, for small positive parameter $\delta$ we define $\Sigma_{\delta}$ as the graph of $U \ni q \mapsto x_{\delta}(q) \in \Omega$ where

$$
x_{\delta}(q):=\delta x(q)-\delta x_{0}+x_{0} .
$$

For example a sphere of radius $R$ originated at $x_{0}$ turns to the sphere of radius $\delta R$ after scaling. Note that equivalence $\left|\partial_{q_{1}} x_{\delta}(q) \times \partial_{q_{1}} x_{\delta}(q)\right|=\delta^{2} \mid \partial_{q_{1}} x(q) \times$ $\partial_{q_{1}} x(q) \mid$ implies the scaling of the surface area $\left|\Sigma_{\delta}\right|=\delta^{2}|\Sigma|$.

\section{Preliminary results for the analysis of poles}

The Birman-Schwinger argument relates the eigenvalues of $H_{\alpha, \beta}$ and zeros of $I-\beta \mathrm{R}_{\alpha, \Sigma \Sigma}(z)$ determined by the condition $\operatorname{ker}\left(I-\beta \mathrm{R}_{\alpha, \Sigma \Sigma}(z)\right) \neq\{0\}$. To recover resonances we show that $\mathrm{R}_{\alpha, \Sigma \Sigma}(z)$ has a second sheet continuation $\mathrm{R}_{\alpha, \Sigma \Sigma}^{I I}(z)$ and the statement

$$
\operatorname{ker}\left(I-\beta \mathrm{R}_{\alpha, \Sigma \Sigma}^{I I}(z)\right) \neq\{0\}
$$

holds for certain $z \in \mathbb{C}_{-}$. 


\subsection{Analytic continuation of $\mathrm{R}_{\alpha, \Sigma \Sigma}(z)$}

We start with the analysis of the first component of $\mathrm{R}_{\alpha, \Sigma \Sigma}(z)$ determined by $\mathrm{R}_{\Sigma \Sigma}(z)$, cf. (3.12). Since $\mathrm{R}_{\Sigma \Sigma}(z)$ is defined by means of the embedding of kernel $\mathcal{G}(z)$, see (2.3), the following lemma will be useful for further discussion.

Lemma 4.1 For any $k \in \mathbb{N}$ the function $\mathcal{G}(z)$ admits the second sheet continuation $\mathcal{G}^{I I}(z)$ through $\left.J_{k}:=\left(k^{2},(k+1)^{2}\right)\right)$ to an open set $\Pi_{k} \subset \mathbb{C}_{-}$and $\partial \Pi_{k} \cap \mathbb{R}=J_{k}$. Moreover, $\mathcal{G}^{I I}(z)$ takes the form

$$
\mathcal{G}^{I I}\left(z ; \underline{x}, \underline{x}^{\prime}, x_{3}, x_{3}^{\prime}\right)=\frac{1}{2 \pi} \sum_{n=1}^{\infty} Z_{0}\left(i \sqrt{z-n^{2}}\left|\underline{x}-\underline{x}^{\prime}\right|\right) \chi_{n}\left(x_{3}\right) \chi_{n}\left(x_{3}^{\prime}\right),
$$

where

$$
Z_{0}\left(i \sqrt{z-n^{2}} \rho\right)= \begin{cases}K_{0}\left(-i \sqrt{z-n^{2}} \rho\right), & \text { for } n>k \\ K_{0}\left(-i \sqrt{z-n^{2}} \rho\right)+i \pi I_{0}\left(i \sqrt{z-n^{2}} \rho\right), & \text { for } n \leq k\end{cases}
$$

and $I_{0}(\cdot)$ standardly denotes the Bessel function.

Proof. The proof is based on edge-of-the-wedge theorem, i.e. our aim is to establish the convergence

$$
\mathcal{G}(\lambda+i 0)=\mathcal{G}^{I I}(\lambda-i 0)
$$

for $\lambda \in J_{k}$. In fact, it suffices to show that the analogous formula holds for $Z_{0}$ and $z=\lambda \pm i 0$.

Assume first that $n>k$. Then $\sqrt{\lambda-n^{2} \pm i 0}=\sqrt{\lambda-n^{2}}$ since $\Im \sqrt{\lambda-n^{2}}>0$. Furthermore, the function $K_{0}(\cdot)$ is analytic in the upper half-plane, consequently, we have $K_{0}\left(-i \sqrt{\lambda-n^{2} \pm i 0} \rho\right)=K_{0}\left(\sqrt{n^{2}-\lambda} \rho\right)$.

Assume now that $n \leq k$. Then $\sqrt{\lambda-n^{2} \pm i 0}= \pm \sqrt{\lambda-n^{2}} \in \mathbb{R}$ which implies

$$
K_{0}\left(-i \sqrt{\lambda-n^{2}+i 0} \rho\right)=K_{0}\left(-i \sqrt{\lambda-n^{2}} \rho\right) .
$$

On the other hand, using the analytic continuation formulae

$$
K_{0}\left(z \mathrm{e}^{m \pi i}\right)=K_{0}(z)-i m \pi I_{0}(z) \quad \text { and } \quad I_{0}\left(z \mathrm{e}^{m \pi i}\right)=I_{0}(z),
$$

for $m \in \mathbb{N}$, we get

$$
\begin{aligned}
Z_{0}\left(\sqrt{\lambda-n^{2}-i 0} \rho\right) & =K_{0}\left(i \sqrt{\lambda-n^{2}} \rho\right)+i \pi I_{0}\left(-i \sqrt{\lambda-n^{2}} \rho\right) \\
& =K_{0}\left(-i \sqrt{\lambda-n^{2}} \rho\right) .
\end{aligned}
$$




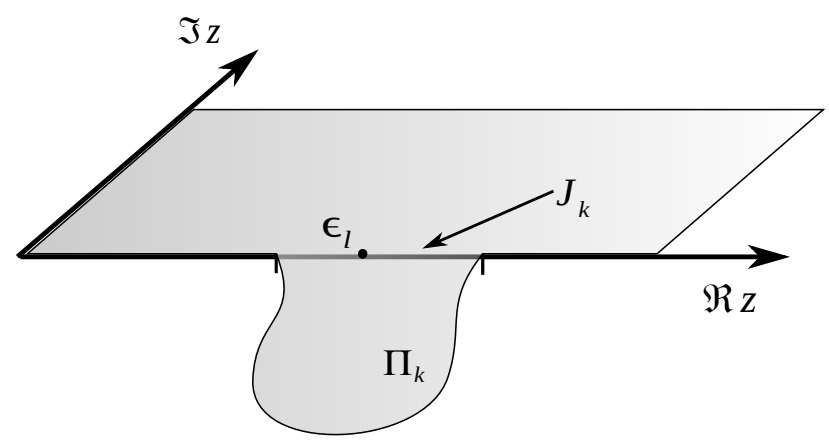

This completes the proof.

The above lemma provides the second sheet continuation of $R(z)$ as well as $\mathrm{R}_{\Sigma \Sigma}(z)$; the latter is defined as the bilateral embedding of $R^{I I}(z)$ to $L^{2}(\Sigma)$.

Remark 4.2 Note that for each $k \in \mathbb{N}$ the analytic continuation of $\mathcal{G}(\cdot)$ through $J_{k}$ leads to different branches. Therefore, we have to keep in mind that the analytic continuation of $\mathcal{G}(\cdot)$ is $k$-dependent.

In the next lemma we show that the operator $\mathrm{R}_{\Sigma_{\delta} \Sigma_{\delta}}^{I I}(z)$ is bounded and derive the operator norm asymptotics if $\delta \rightarrow 0$.

Lemma 4.3 Assume that $k \in \mathbb{N}$ and $\lambda \in J_{k}$. Let $z=\lambda-i \varepsilon$, where $\varepsilon$ is a small positive number. Operator $\mathrm{R}_{\Sigma_{\delta} \Sigma_{\delta}}^{I I}(z)$ is bounded and its norm admits the asymptotics

$$
\left\|\mathrm{R}_{\Sigma_{\delta} \Sigma_{\delta}}^{I I}(z)\right\|=o(1)
$$

where the error term is understood with respect to $\delta$.

Proof. To estimate the kernel of $\mathrm{R}_{\Sigma_{\delta} \Sigma_{\delta}}^{I I}(z)$ we use 4.19 , i.e.

$$
\begin{aligned}
\mathcal{G}_{\Sigma_{\delta} \Sigma_{\delta}}^{I I}\left(z ; \rho, x_{3}, x_{3}^{\prime}\right)= & \frac{1}{2 \pi}\left(\sum_{n=1}^{\infty} K_{0}\left(\kappa_{n}(z) \rho\right) \chi_{n}\left(x_{3}\right) \chi_{n}\left(x_{3}^{\prime}\right)\right. \\
& \left.+\sum_{n \leq k} I_{0}\left(-\kappa_{n}(z) \rho\right) \chi_{n}\left(x_{3}\right) \chi_{n}\left(x_{3}^{\prime}\right)\right)
\end{aligned}
$$

where

$$
\rho=\left|\underline{x}-\underline{x}^{\prime}\right|, \quad \text { and } \quad x, x^{\prime} \in \Sigma_{\delta} .
$$


First, we consider 4.23. The expression

$$
\left|I_{0}\left(-\kappa_{n}(z) \rho\right) \chi_{n}(\cdot) \chi_{n}(\cdot)\right|
$$

is bounded. Therefore the operator defined by the kernel (4.23) is also bounded and the corresponding operator norm in $L^{2}\left(\Sigma_{\delta}\right)$ behaves as $\left|\Sigma_{\delta}\right|^{2}=$ $\mathcal{O}\left(\delta^{4}\right)$.

The analysis of the term 4.22 is more involving because it consists of infinite number of components. The asymptotics:

$$
K_{0}\left(\kappa_{n}(z) \rho\right)-K_{0}(n \rho)=\ln \sqrt{1-\frac{z}{n^{2}}}(1+\mathcal{O}(\rho))
$$

implies

$$
\sum_{n=1}^{\infty}\left|\left(K_{0}\left(\kappa_{n}(z) \rho\right)-K_{0}(n \rho)\right) \chi_{n}\left(x_{3}\right) \chi_{n}\left(x_{3}^{\prime}\right)\right|=C+\mathcal{O}(\rho) ;
$$

remind that $\kappa_{n}(z)$ is defined by $(2.4)$. To estimate $\sum_{n=1}^{\infty} K_{0}(n \rho) \chi_{n}\left(x_{3}\right) \chi_{n}\left(x_{3}^{\prime}\right)$ we borrow the idea from [15] and use [26, Chap. 10, II, 5.9.1.4.] to get

$$
\begin{aligned}
\sum_{n=1}^{\infty} K_{0}(n \rho) \cos (n a)= & \frac{\pi}{2 \sqrt{\rho^{2}+a^{2}}}+\frac{1}{2}\left(\ln \frac{\rho}{4 \pi}-\psi(1)\right) \\
& +\frac{\pi}{2} \sum_{n=1}^{\infty}\left(\frac{1}{\sqrt{(2 n \pi+a)^{2}+\rho^{2}}}-\frac{1}{2 n \pi}\right) \\
& +\frac{\pi}{2} \sum_{n=1}^{\infty}\left(\frac{1}{\sqrt{(2 n \pi-a)^{2}+\rho^{2}}}-\frac{1}{2 n \pi}\right) .
\end{aligned}
$$

For $x, x^{\prime} \in \Sigma_{\delta}$ the terms 4.26 and 4.27 can be majorized by $C\left(\sum_{n=1}^{\infty} \frac{1}{n^{2}}\right)$, i.e. by a uniform constant. Consequently, using the above estimates together with the equivalence $\sin a \sin b=\frac{1}{2}(\cos (a-b)-\cos (a+b))$ we get after straightforward calculations

$$
\left|\sum_{n=1}^{\infty} K_{0}\left(\kappa_{n}(z) \rho\right) \chi_{n}\left(x_{3}\right) \chi_{n}\left(x_{3}^{\prime}\right)\right| \leq C\left(\frac{1}{\left|x-x^{\prime}\right|}+\ln \left|\underline{x}-\underline{x}^{\prime}\right|\right) ;
$$

the singular terms in the above estimates come from (4.25). Let us analyze the left hand side of 4.28). First, we consider the component $\mathcal{P}\left(x, x^{\prime}\right):=$ 
$\frac{1}{\left|x-x^{\prime}\right|}$ which gives

$$
\int_{\Sigma_{\delta}} \mathcal{P}\left(x, x^{\prime}\right) \mathrm{d} \Sigma_{\delta}=\left(\mathcal{P} * \delta_{\Sigma_{\delta}}\right)(x)=\int_{\Sigma_{\delta}} \frac{1}{\left|x-x^{\prime}\right|} \mathrm{d} \Sigma_{\delta} .
$$

To conclude the desired convergence we employ the concept of generalized Kato measure. Namely, since the Dirac delta on $\Sigma_{\delta}$ defines Kato measure we obtain

$$
\sup _{x \in \Sigma_{\delta}} \int_{\Sigma_{\delta}} \mathcal{P}\left(x, x^{\prime}\right) \mathrm{d} \Sigma_{\delta}=o(1),
$$

where the right hand side asymptotics is understood in the sense of convergence with respect to $\delta$. Employing the Schur argument we conclude that the norm of the integral operator with the kernel $\mathcal{P}\left(x, x^{\prime}\right)$ acting from $L^{2}\left(\Sigma_{\delta}\right)$ to $L^{2}\left(\Sigma_{\delta}\right)$ behaves as $o(1)$. The term $\ln \left|\underline{x}-\underline{x}^{\prime}\right|$ contributing to 4.28 can be estimated in the analogous way.

To recover the second sheet continuation of $\mathrm{R}_{\alpha, \Sigma \Sigma}(\cdot)$ it remains to construct the analytic extensions of $\omega_{n}(z)$ and $\Gamma_{n}(z)$, cf. (3.12).

Lemma 4.4 Given $n \in \mathbb{N}$ the functions $\omega_{n}(z)$ and $\Gamma_{n}(z)$ admit the second sheet continuations $\omega_{n}^{I I}(z)$ and $\Gamma_{n}^{I I}(z)$ to $\Pi_{k}$ through $J_{k}=\left(k^{2},(k+1)^{2}\right), k \in \mathbb{N}$ defined by

$$
\omega_{n}^{I I}(z ; x):=\frac{1}{2 \pi} Z_{0}\left(i \sqrt{z-n^{2}}|\underline{x}|\right) \chi_{n}\left(x_{3}\right),
$$

where $Z_{0}$ is determined by (4.19), and

$$
\Gamma_{n}^{I I}(z)=\left\{\begin{array}{lr}
\frac{1}{2 \pi}\left(2 \pi \alpha-\psi(1)+\ln \frac{\sqrt{z-n^{2}}}{2 i}\right), & \text { for } \quad n>k \\
\frac{1}{2 \pi}\left(2 \pi \alpha-\psi(1)+\ln \frac{\sqrt{z-n^{2}}}{2 i}-\pi i\right), & \text { for } \quad n \leq k .
\end{array}\right.
$$

Proof. The construction of $\omega_{n}^{I I}(z)$ can be obtained mimicking the arguments from the proof of Lemma 4.1 .

To get $\Gamma^{I I}(z)$ we first assume $k<n$ and $z=\lambda \pm i \varepsilon, \lambda \in\left(k^{2},(k+1)^{2}\right)$. Then $\ln \frac{\sqrt{\lambda-n^{2} \pm i 0}}{i}=\ln \sqrt{n^{2}-\lambda}$ and, consequently, $\Gamma_{n}(\lambda+i 0)=\Gamma_{n}^{I I}(\lambda-i 0)$.

Assume now that $n \leq k$. Then we have $\lambda-n^{2}>0$ and $\ln \frac{\sqrt{\lambda-n^{2} \pm i 0}}{i}=$ $\ln \sqrt{\lambda-n^{2} \pm i 0} \mp \frac{\pi}{2} i$ which implies

$$
\Gamma_{n}(\lambda+i 0)=\Gamma_{n}^{I I}(\lambda-i 0)=\frac{1}{2 \pi}\left(2 \pi \alpha-\psi(1)+\ln \sqrt{\lambda-n^{2}}-\frac{\pi}{2} i\right) .
$$


This, in view of edge-of-the-wedge theorem, completes the proof.

Henceforth, we assume that $\epsilon_{n} \neq k^{2}$ for any $k, n \in \mathbb{N}$. Suppose $z=\lambda-i \varepsilon$, where $\varepsilon$ is a small non-negative number and $\lambda \in J_{k}$. At most one eigenvalue $\epsilon_{l}$ can exist in the interval $J_{k}$. Assuming that $z \in\left(\Pi_{k} \cup J_{k}\right) \backslash \epsilon_{l}$ we define the analytic functions $z \mapsto \Gamma_{n}^{I I}(z)^{-1}$ for $n \in \mathbb{N}$. Then the second sheet continuation of the resolvent takes the form

$$
\mathrm{R}_{\alpha, \Sigma \Sigma}^{I I}(z)=\mathrm{R}_{\Sigma \Sigma}^{I I}(z)+\sum_{n=1}^{\infty} \Gamma_{n}^{I I}(z)^{-1}\left(w_{n}^{I I}(\bar{z}), \cdot\right)_{\Sigma} w_{n}^{I I}(z)
$$

for $z \in\left(\Pi_{k} \cup J_{k}\right) \backslash \epsilon_{l}$.

Notation. In the following we will avoid the superscript II keeping in mind that all quantities depending on $z$ are defined for second sheet continuation if $\Im z<0$ which admits infinitely many branches $\Pi_{k}, k \in \mathbb{N}$.

Assume that $\epsilon_{l} \in J_{k}$. Having in mind latter purposes we define

$$
A_{l}(z):=\sum_{n \neq l} \Gamma_{n}(z)^{-1}\left(w_{n}(\bar{z}), \cdot\right)_{\Sigma_{\delta}} w_{n}(z)
$$

for $z \in\left(\Pi_{k} \cup J_{k}\right) \backslash \epsilon_{l}$. The following lemma states the operator norm asymptotics.

Lemma 4.5 Operator $A_{l}(z): L^{2}\left(\Sigma_{\delta}\right) \rightarrow L^{2}\left(\Sigma_{\delta}\right)$ is bounded and the operator norm satisfies

$$
\left\|A_{l}(z)\right\| \leq C\left|\Sigma_{\delta}\right|
$$

Proof. Suppose that $z=\lambda-i \varepsilon$. We derive the estimates

$$
\begin{aligned}
\left|\left(A_{l}(z) f, f\right)_{\Sigma_{\delta}}\right| \leq & \left(\sum_{n \neq l}\left|\Gamma_{n}(z)^{-1}\right|\left\|w_{n}(z)\right\|_{\Sigma_{\delta}}^{2}\right)\|f\|_{\Sigma_{\delta}}^{2} \leq \\
& C\left(\sum_{n \neq l}\left\|w_{n}(z)\right\|_{\Sigma_{\delta}}^{2}\right)\|f\|_{\Sigma_{\delta}}^{2} ;
\end{aligned}
$$

to obtain 4.35) we use 4.30. Now our aim is to show

$$
\sum_{n \neq l}\left\|w_{n}(z)\right\|_{\Sigma_{\delta}}^{2} \leq C\left|\Sigma_{\delta}\right|
$$


To find a bound for the left hand side of 4.36) we analyse first the behaviour of $w_{n}(z)$ for large $n$ and $z \in\left(\Pi_{k} \cup J_{k}\right) \backslash \epsilon_{l}$. For this aim we employ (4.29) and (4.19). Note that for $n>k$ function $w_{n}(z)$ admits the representation:

$$
w_{n}(z, x)=\frac{1}{2 \pi} K_{0}\left(\kappa_{n}(z)|\underline{x}|\right) \chi_{n}\left(x_{3}\right),
$$

where $x \in \Sigma_{\delta}$. Using again the large argument expansion 3.15 and the fact that $\Re\left(-i \sqrt{z-n^{2}}\right) \sim n$ we get the estimate

$$
\left|w_{n}(z, x)\right| \leq C \mathrm{e}^{-r_{\min } n},
$$

where $r_{\min }=\min _{x \in \Sigma_{\delta}}|\underline{x}|$. This implies

$$
\sum_{n>k, n \neq l}\left\|w_{n}(z)\right\|_{\Sigma_{\delta}}^{2} \leq C\left|\Sigma_{\delta}\right|=\mathcal{O}\left(\delta^{2}\right)
$$

On the other hand for $n \leq k$ function $w_{n}(z)$ consists of $K_{0}$ and $I_{0}$, see 4.19). Both functions are continuous on $\Sigma_{\delta}$ and therefore $\left\|w_{n}\right\|_{\Sigma_{\delta}}^{2} \leq C\left|\Sigma_{\delta}\right|$. Since the number of such components is finite, in view of (4.37), we come to 4.36) which completes the proof.

\section{Complex poles of resovent}

Assume that $\epsilon_{l} \in J_{k}$. Suppose that $\delta$ is sufficiently small. It follows from Lemmae 4.3 and 4.5 that the operators $I-\beta \mathrm{R}_{\Sigma_{\delta} \Sigma_{\delta}}(z)$ and $I-\beta A_{l}(z)$ acting in $L^{2}\left(\Sigma_{\delta}\right)$ are invertible for $z \in\left(\Pi_{k} \cup J_{k}\right) \backslash \epsilon_{l}$ and it makes sense to introduce auxiliary notation

$$
G_{\Sigma_{\delta}}(z):=\left(I-\beta \mathrm{R}_{\Sigma_{\delta} \Sigma_{\delta}}(z)\right)^{-1} .
$$

Since the norm of $G_{\Sigma_{\delta}}(z) A_{l}(z): L^{2}\left(\Sigma_{\delta}\right) \rightarrow L^{2}\left(\Sigma_{\delta}\right)$ tends to 0 if $\delta \rightarrow 0$ therefore the operator $I+\beta G_{\Sigma_{\delta}}(z) A_{l}(z)$ is invertible as well.

The following theorem "transfer" the analysis of resonances from the operator equation to the complex valued function equation.

Theorem 5.1 Suppose $\epsilon_{l} \in J_{k}$ and assume that $z \in\left(\Pi_{k} \cup J_{k}\right) \backslash \epsilon_{l}$. Then the condition

$$
\operatorname{ker}\left(I-\beta \mathrm{R}_{\alpha, \Sigma_{\delta} \Sigma_{\delta}}(z)\right) \neq\{0\}
$$

is equivalent to

$$
\Gamma_{l}(z)+\beta\left(w_{l}(\bar{z}), T_{l}(z) w_{l}(z)\right)_{\Sigma_{\delta}}=0
$$

where

$$
T_{l}(z):=\left(I-\beta G_{\Sigma_{\delta}}(z) A_{l}(z)\right)^{-1} G_{\Sigma_{\delta}}(z) .
$$


Proof. The strategy of the proof is partially based on the idea borrowed from [9]. The following equivalences

$$
\begin{aligned}
I-\beta \mathrm{R}_{\alpha, \Sigma_{\delta} \Sigma_{\delta}}(z)= & \\
= & \left(I-\beta \mathrm{R}_{\Sigma_{\delta} \Sigma_{\delta}}(z)\right)\left(I-\beta G_{\Sigma_{\delta}}(z) A_{l}(z)\right. \\
& \left.-\beta \Gamma_{l}(z)^{-1}\left(w_{l}(\bar{z}), \cdot\right)_{\Sigma_{\delta}} G_{\Sigma_{\delta}}(z) w_{l}(z)\right) \\
= & \left(I-\beta \mathrm{R}_{\Sigma_{\delta} \Sigma_{\delta}}(z)\right)\left(I-\beta G_{\Sigma_{\delta}}(z) A_{l}(z)\right) \times \\
& {\left[I-\beta \Gamma_{l}(z)^{-1}\left(w_{l}(\bar{z}), \cdot\right)_{\Sigma_{\delta}} T_{l}(z) w_{l}(z)\right], }
\end{aligned}
$$

show that 5.38 is equivalent to

$$
\operatorname{ker}\left[I-\beta \Gamma_{l}(z)^{-1}\left(w_{l}(\bar{z}), \cdot\right)_{\Sigma_{\delta}} T_{l}(z) w_{l}(z)\right] \neq\{0\} .
$$

The above condition is formulated for a rank one operator and, consequently, it is equivalent to 5.39 .

Theorem 5.1 shows that the problem of complex poles of resolvent $R_{\alpha, \beta}(z)$ can be shifted to the problem of the roots analysis of

$$
\eta_{l}(z, \delta)=0, \quad \text { where } \quad \eta_{l}(z, \delta):=\Gamma_{l}(z)-\beta \vartheta_{l}(z, \delta)
$$

and

$$
\vartheta_{l}(z, \delta):=\left(w_{l}(\bar{z}), T_{l}(z) w_{l}(z)\right)_{\Sigma_{\delta}} .
$$

The further discussion is devoted to figuring out roots of $(5.40)$. In the following we apply the expansion $(1+A)^{-1}=\left(1-A+A^{2}-A^{3} \ldots\right)$ valid if $\|A\|<1$. Taking $-\beta \mathrm{R}_{\Sigma_{\delta} \Sigma_{\delta}}(z)$ as $A$ we get

$$
G_{\Sigma_{\delta}}(z)=\left(I-\beta \mathrm{R}_{\Sigma_{\delta} \Sigma_{\delta}}(z)\right)^{-1}=I+\breve{\mathrm{R}}(z), \quad \breve{\mathrm{R}}(z):=\sum_{n=1}\left(\beta \mathrm{R}_{\Sigma_{\delta} \Sigma_{\delta}}(z)\right)^{n} .
$$

Expanding the analogous sum for $-\beta G_{\Sigma_{\delta}}(z) A_{l}(z)$ one obtains

$$
\left(I-\beta G_{\Sigma_{\delta}}(z) A_{l}(z)\right)^{-1}=I+\beta A_{l}(z)+\beta \breve{\mathrm{R}}(z) A_{l}(z)+\ldots
$$

In view of Lemmae 4.5 and 4.3 the norm of $\mathrm{R}_{\Sigma_{\delta} \Sigma_{\delta}}(z) A_{l}(z)$ behaves as $o(1)\left\|A_{l}(z)\right\|_{\Sigma_{\delta}}$ for $\delta \rightarrow 0$ and the same asymptotics holds for the operator norm of $\breve{\mathrm{R}}(z) A_{l}(z)$. The further terms in $(5.42)$ are of smaller order with respect to $\delta$. Consequently, applying again (5.42) we conclude that $T_{l}(z)$ admits the following expansion

$$
T_{l}(z)=I+\beta A_{l}(z)+\breve{\mathrm{R}}(z)+\ldots
$$

Using the above statements we can formulate the main result. 
Theorem 5.2 Suppose that $\epsilon_{l} \in J_{k}$ and consider the function $\eta_{l}(z, \delta): \Pi_{k} \cup$ $J_{k} \times\left[0, \delta_{0}\right) \rightarrow \mathbb{C}$, where $\delta_{0}>0$, defined by (5.40). Then the equation

$$
\eta_{l}(z, \delta)=0
$$

possesses a solution which is determined by the function $\delta \mapsto z(\delta) \in \mathbb{C}$ with the following asymptotics

$$
z_{l}(\delta)=\epsilon_{l}+\mu_{l}(\delta), \quad\left|\mu_{l}(\delta)\right|=o(1) .
$$

Moreover, the lowest order term of $\mu_{l}(\cdot)$ takes the form

$$
\begin{aligned}
\mu_{l}(\delta)=\quad & 4 \pi \xi_{\alpha} \beta\left\{\left\|w_{l}\left(\epsilon_{l}\right)\right\|_{\Sigma_{\delta}}^{2}\right. \\
& +\beta \sum_{n \neq l} \Gamma_{n}\left(\epsilon_{l}\right)^{-1}\left|\left(w_{l}\left(\epsilon_{l}\right), w_{n}\left(\epsilon_{l}\right)\right)_{\Sigma_{\delta}}\right|^{2} \\
& \left.+\left(w_{l}\left(\epsilon_{l}\right), \breve{\mathrm{R}}\left(\epsilon_{l}\right) w_{l}\left(\epsilon_{l}\right)\right)_{\Sigma_{\delta}}\right\}
\end{aligned}
$$

Proof. Note that $z \mapsto \eta_{l}(z, \delta)$, cf. (5.40), is analytic and $\eta_{l}\left(\epsilon_{l}, 0\right)=0$. Using 4.30 one obtains

$$
\left.\frac{d \Gamma_{n}(z)}{d z}\right|_{z=\epsilon_{n}}=\frac{1}{4 \pi \xi_{\alpha}}<0, \quad n \in \mathbb{N} .
$$

Combining this with

$$
\left.\frac{\partial \vartheta_{n}(z, \delta)}{\partial z}\right|_{z=\epsilon_{l}, \delta=0}=0
$$

we get $\left.\frac{\partial \eta_{l}}{\partial z}\right|_{\delta=0}=\frac{1}{4 \pi \xi_{\alpha}} \neq 0$. In view of the Implicit Function Theorem we conclude that the equation (5.40) admits a unique solution which a continuous function of $\delta \mapsto z_{l}(\delta)$ and $z_{l}(\delta)=\epsilon_{l}+o(1)$. To reconstruct asymptotics of $z(\cdot)$ first we expand $\Gamma_{l}(z)$ into the Taylor sum

$$
\Gamma_{l}(z)=\frac{1}{4 \pi \xi_{\alpha}}\left(z-\epsilon_{l}\right)+\mathcal{O}\left(\left(z-\epsilon_{l}\right)^{2}\right) .
$$

Then the spectral equation 5.40 reads

$$
z=\epsilon_{l}+4 \pi \xi_{\alpha} \beta \vartheta_{l}(z, \delta)+\mathcal{O}\left(\left(z-\epsilon_{l}\right)^{2}\right) .
$$


Now we expand $\vartheta_{l}(z, \delta)$. Using (5.43) and 4.33) we reconstruct its first order term which reads

$$
\begin{aligned}
& \left\{\left\|w_{l}\left(\epsilon_{l}\right)\right\|_{\Sigma_{\delta}}^{2}+\beta \sum_{n \neq l} \Gamma_{n}\left(\epsilon_{l}\right)^{-1}\left|\left(w_{l}\left(\epsilon_{l}\right), w_{n}\left(\epsilon_{l}\right)\right)_{\Sigma_{\delta}}\right|^{2}\right. \\
& \left.+\left(w_{l}\left(\epsilon_{l}\right), \breve{\mathrm{R}}\left(\epsilon_{l}\right) w_{l}\left(\epsilon_{l}\right)\right)_{\Sigma_{\delta}}\right\} .
\end{aligned}
$$

Applying the asymptotics $z_{l}\left(\epsilon_{l}\right)=\epsilon_{l}+o(\delta)$ and the fact that $\vartheta_{l}(\cdot, \cdot)$ is analytic with respect to complex variable we get formula for $\mu(\cdot)$.

\subsection{Analysis of imaginary part of the pole}

Since the imaginary component of resonance pole has a physical meaning we dedicate to this problem a special discussion. The information on the lowest order term of the pole imaginary component is contained in (5.47) and (5.48). On the other hand, note that only the components subscripted by $n \leq k$ admit a non-zero imaginary parts. Therefore

$$
\begin{aligned}
\Im & \left(4 \pi \xi _ { \alpha } \beta \left(\beta \sum_{n \leq k} \Gamma_{n}\left(\epsilon_{l}\right)^{-1}\left|\left(w_{l}\left(\epsilon_{l}\right), w_{n}\left(\epsilon_{l}\right)\right)_{\Sigma_{\delta}}\right|^{2}\right.\right. \\
& \left.\left.+\left(w_{l}\left(\epsilon_{l}\right), \breve{\mathrm{R}}\left(\epsilon_{l}\right) w_{l}\left(\epsilon_{l}\right)\right)_{\Sigma_{\delta}}\right)\right) .
\end{aligned}
$$

determines the lowest order term of $\Im \mu(\delta)$.

Sign and asymptotics of $\Im \mu(\delta)$ with respect to $\Sigma_{\delta}$. Recall that $\epsilon_{l} \in J_{k}$. First we analyse (5.49) and for this aim we define

$$
\iota_{l, n}:=\frac{1}{2 \pi}\left(2 \pi \alpha+\ln \frac{\sqrt{\epsilon_{l}-n^{2}}}{2}-\psi(1)\right),
$$

for $n \leq k$. Relying on 4.31 we get

$$
\Gamma_{l}\left(\epsilon_{l}\right)^{-1}=\frac{1}{\iota_{l, n}^{2}+(1 / 2)^{2}}\left(\iota_{l, n}+\frac{1}{2} i\right)
$$

if $n \leq k$. Consequently, formula $(5.49)$ is equivalent to

$$
\Im 4 \pi \xi_{\alpha} \beta^{2} \sum_{n \leq k} \frac{1}{2} \frac{1}{\iota_{l, n}^{2}+(1 / 2)^{2}}\left|\left(w_{l}\left(\epsilon_{l}\right), w_{n}\left(\epsilon_{l}\right)\right)_{\Sigma_{\delta}}\right|^{2} .
$$


The above expression is negative because $\xi_{\alpha}<0$. Moreover, since both $w_{l}\left(\epsilon_{l}\right)$ and $w_{l}\left(\epsilon_{n}\right)$ are continuous in $\Omega \backslash I$ we have $\left|\left(w_{l}\left(\epsilon_{l}\right), w_{n}\left(\epsilon_{l}\right)\right)_{\Sigma_{\delta}}\right|^{2} \sim\left|\Sigma_{\delta}\right|^{2}$. This means that (5.49) behaves as $\mathcal{O}\left(\left|\Sigma_{\delta}\right|^{2}\right)$. To recover the asymptotics of $(5.50)$ we restrict ourselves to the lowest order term of $\breve{R}(z)$, cf. $(5.43)$, namely

$$
v_{l}:=\Im 4 \pi \xi_{\alpha} \beta^{2}\left(w_{l}\left(\epsilon_{l}\right), \mathrm{R}_{\Sigma_{\delta} \Sigma_{\delta}}\left(\epsilon_{l}\right) w_{l}\left(\epsilon_{l}\right)\right)_{\Sigma_{\delta}} .
$$

Using analytic continuation formulae 4.20 and employing the small argument expansion, cf. [2],

$$
K_{0}(z) \sim-\ln z
$$

where $-\pi<\arg z<\pi$ states the plane cut for the logarithmic function, one gets

$$
v_{l} \sim \Im \pi \xi_{\alpha} \beta^{2} \sum_{n \leq k}\left(\int_{\Sigma_{\delta}} w_{l}\left(\epsilon_{l}\right) \chi_{n}\right)^{2}=\mathcal{O}\left(\left|\Sigma_{\delta}\right|^{2}\right)
$$

One can easily see that $v_{l}<0$. Summing up the above discussion we can formulate the following conclusion.

Proposition 5.3 The resonance pole takes the form $z_{l}(\delta)=\epsilon_{l}+\mu(\delta)$ with the lowest order of $\Im \mu(\delta)$ given by

$$
\pi \xi_{\alpha} \beta^{2} \sum_{n \leq k}\left(\frac{2}{\iota_{l, n}^{2}+(1 / 2)^{2}}\left|\left(w_{l}\left(\epsilon_{l}\right), w_{n}\left(\epsilon_{l}\right)\right)_{\Sigma_{\delta}}\right|^{2}+\left(\int_{\Sigma_{\delta}} w_{l}\left(\epsilon_{l}\right) \chi_{n}\right)^{2}\right) .
$$

It follows from the above formula that $\Im \mu(\delta)<0$ and the asymptotics

$$
\Im \mu(\delta)=\mathcal{O}\left(\left|\Sigma_{\delta}\right|^{2}\right)
$$

holds. Moreover, the lowest order of $\Im \mu(\delta)$ is independent of sign of $\beta$.

Note that for the special geometrical cases the embedded eigenvalues can survive after introducing $\Sigma_{\delta}$ since the "perturbed" eigenfunctions are not affected by presence of $\Sigma_{\delta}$. Let us consider

$$
\Pi_{l}:=\left\{x \in \Omega: x=\left(\underline{x}, \frac{\pi}{l}\right), \quad l \in \mathbb{N}\right.
$$

and assume that $\Sigma_{\delta} \subset \Pi_{l}$. Then $w_{m l}(z)=0$ for each $m \in \mathbb{N}$ and, consequently $\vartheta_{m l}(z, \delta)=0$, cf. (5.40). This implies the following statement.

Proposition 5.4 Suppose that $\Sigma \subset \Pi_{l}$. Then for all $m \in \mathbb{N}$ the numbers $\epsilon_{m l}$ remain the embedded eigenvalues of $H_{\alpha, \beta}$. 


\section{Acknowledgements}

The author thanks the referees for reading the paper carefully, removing errors and recommending various improvements in exposition.

The work was supported by the project DEC-2013/11/B/ST1/03067 of the Polish National Science Centre.

\section{References}

[1] S. Albeverio, F. Gesztesy, R. Høegh-Krohn, H. Holden: Solvable Models in Quantum Mechanics, 2nd printing (with Appendix by P. Exner), AMS, Providence, R.I., 2004.

[2] M. Abramowitz and I. Stegun: Handbook of Mathematical Functions, 1972.

[3] L. M. Baskin, B. A. Plamenevskii, O. V. Sarafanov: Effect of magnetic field on resonant tunneling in 3D waveguides of variable cross-section $J$. Math. Sci. 196 (4) (2013) 469-489.

[4] L. M. Baskin, M. Kabardov, P. Neittaanmäki, B. A. Plamenevskii, O. V. Sarafanov: Asymptotic and numerical study of resonant tunneling in two-dimensional quantum waveguides of variable cross section, Computational Mathematics and Mathematical Physics 53 (11) (2013), 16641683.

[5] J. Behrndt, P. Exner, M. Holzmann, V. Lotoreichik: Approximation of Schrödinger operators with $\delta$-interactions supported on hypersurfaces, Math. Nachr. (2016), 1-34.

[6] D. Borisov, P. Exner, A. Golovina: Tunneling resonances in systems without a classical trapping, J. Math. Phys. 54, 012102 (2013).

[7] J.F. Brasche, P. Exner, Yu.A. Kuperin, P. Šeba: Schrödinger operators with singular interactions, J. Math. Anal. Appl. 184 (1994), 112-139.

[8] P. Briet, M. Gharsalli: Stark resonances in 2-dimensional curved quantum waveguides Rep. Math. Phys. 76 (3), (2015) 317-338.

[9] Yu.P. Chuburin: Perturbation Theory of Resonances and Embedded Eigenvalues of the Schrodinger Operator For a Crystal Film Teoret. Mat. Fiz. 1433 (2005), 417430.

[10] A. L. Delitsyn, B-T. Nguyen, D. S. Grebenkov: Trapped modes in finite quantum waveguides The European Physical Journal B-Condensed Matter and Complex Systems 856 (2012), 1-12. 
[11] P. Exner, S. Kondej: Curvature-induced bound states for a $\delta$ interaction supported by a curve in $\mathbb{R}^{3}$, Ann. H. Poincaré 3 (2002), 967-981.

[12] P. Exner, S. Kondej: Schrödinger operators with singular interactions: a model of tunneling resonances Journal of Physics A : Mathematical and General 37 (2004), 8255-8277.

[13] P. Exner, H. Kovařík, Quantum wavequides, Springer 2015.

[14] P. Exner, D. Krejčiř́k: Quantum waveguides with a lateral semitransparent barrier: spectral and scattering properties Journal of Physics A: Mathematical and General 32 (1999), 4475-4494.

[15] P. Exner, K. Nemcová: Quantum mechanics of layers with a finite number of point perturbation, Journal of Physics A: Mathematical and General 43(3) (2002), 1152-1184.

[16] S. V. Frolov, I. Yu. Popov: Resonances for laterally coupled quantum waveguides, Journal of Mathematical Physics 41 (2000), 4391-4405.

[17] S. V. Frolov, I. Yu. Popov: Three laterally coupled quantum waveguides: breaking of symmetry and resonance asymptotics, Journal of Physics A: Mathematical and General 36(6) (2003).

[18] T. Kato: Pertubation theory for linear operators, Springer-Verlag Berlin Heidelberg New York 1980.

[19] S. Kondej: Resonances induced by broken symmetry in a system with a singular potential, Ann. Henri Poincaré 13 (2012)

[20] S. Kondej, D. Krejčiř́ik: Spectral analysis of a quantum system with a double line singular interaction Publ. RIMS, Kyoto University 49 (2013), 831-859.

[21] S. Kondej, W. Leoński: Mathematical and Theoretical Journal of Physics A 47(22) (2014), 1416-1438.

[22] H. Kovařík, A. Sacchetti: Resonances in twisted quantum waveguides, J. Phys. A 40 (2007) 83718384.

[23] J.L. Lions, E. Magenes: Non-Homogeneous Boundary Value Problems and Applications, vol. I, Springer, Heidelberg 1972.

[24] A. Posilicano: A Krein-like Formula for Singular Perturbations of SelfAdjoint Operators and Applications, J. Funct. Anal. 183 (2001), 109147.

[25] A. Posilicano: Boundary triples and Weyls function for singular perturbations of self-adjoint operators, Meth. Fun. Anal. Top. 10 (2) (2004), $57-63$. 
[26] A. P. Prudnikov Y. O. Brychkov, O. I. Marichev, Integraly i rady, I.Elementarnye funkcii, II. Specialnye funkcii, III. Nauka Moskva. 19811983. 